\title{
Exact cosmological solutions with nonminimal derivative coupling
}

\author{
Sergey V. Sushkov \\ Department of General Relativity and Gravitation, Kazan State University, \\ Kremlevskaya str. 18, Kazan 420008, Russia and \\ Department of Mathematics, Tatar State University of Humanities \\ and Education, Tatarstan str. 2, Kazan 420021, Russia
}

\begin{abstract}
We consider a gravitational theory of a scalar field $\phi$ with nonminimal derivative coupling to curvature. The coupling terms have the form $\kappa_{1} R \phi_{, \mu} \phi^{, \mu}$ and $\kappa_{2} R_{\mu \nu} \phi^{, \mu} \phi^{, \nu}$ where $\kappa_{1}$ and $\kappa_{2}$ are coupling parameters with dimensions of length-squared. In general, field equations of the theory contain third derivatives of $g_{\mu \nu}$ and $\phi$. However, in the case $-2 \kappa_{1}=\kappa_{2} \equiv \kappa$ the derivative coupling term reads $\kappa G_{\mu \nu} \phi^{, \mu} \phi^{, \nu}$ and the order of corresponding field equations is reduced up to second one. Assuming $-2 \kappa_{1}=\kappa_{2}$, we study the spatially-flat Friedman-Robertson-Walker model with a scale factor $a(t)$ and find new exact cosmological solutions. It is shown that properties of the model at early stages crucially depends on the sign of $\kappa$. For negative $\kappa$ the model has an initial cosmological singularity, i.e. $a(t) \sim\left(t-t_{i}\right)^{2 / 3}$ in the limit $t \rightarrow t_{i}$; and for positive $\kappa$ the universe at early stages has the quasi-de Sitter behavior, i.e. $a(t) \sim e^{H t}$ in the limit $t \rightarrow-\infty$, where $H=(3 \sqrt{\kappa})^{-1}$. The corresponding scalar field $\phi$ is exponentially growing at $t \rightarrow-\infty$, i.e. $\phi(t) \sim e^{-t / \sqrt{\kappa}}$. At late stages the universe evolution does not depend on $\kappa$ at all; namely, for any $\kappa$ one has $a(t) \sim t^{1 / 3}$ at $t \rightarrow \infty$. Summarizing, we conclude that a cosmological model with nonminimal derivative coupling of the form $\kappa G_{\mu \nu} \phi^{, \mu} \phi^{, \nu}$ is able to explain in a unique manner both a quasi-de Sitter phase and an exit from it without any fine-tuned potential.
\end{abstract}

PACS numbers: $98.80 . \mathrm{Cq}$ 04.20.Jb

\section{INTRODUCTION}

For many years scalar fields have been an object of great interest for physicists. The reasons for this are manifold. One of them is quite pragmatic: models with scalar fields are relatively simple, and therefore it appeared possible to study them in detail and then extrapolate the results to more realistic and complicated models. More physical motivations include such important topics as the idea about variable "fundamental" constants, the Jordan-Brans-Dicke theory initially elaborated to solve the Mach problem, the Kaluza-Klein compactification scheme, the low-energy limit of the superstring theory, and others. Scalar fields play an especially important role in cosmology. As a bright example, one may mention numerous inflationary models in which inflation in the early Universe is typically driven by a fundamental scalar field called an inflaton. Furthermore, a recent discovery of cosmic acceleration has only refreshed the interest to scalar fields which began to be considered as candidates to explain dark energy phenomena.

The rather general form of action for a scalar-tensor theory of gravity with a single scalar field $\phi$ can be given as $^{1}$

$$
S=\int d^{4} x \sqrt{-g}\left\{\frac{1}{8 \pi} F(\phi, R)-h(\phi) g_{\mu \nu} \phi^{, \mu} \phi^{, \nu}\right\},
$$

where $g_{\mu \nu}$ is a metric, $g=\operatorname{det}\left(g_{\mu \nu}\right)$, and $R$ is the scalar curvature. Functions $F(\phi, R)$ and $h(\phi)$ are varying from theory to theory. The function $h(\phi)$ is responsible for the sign of kinetic energy of the scalar field. For example, the choice $h(\phi) \equiv-1$ leads to a wide class of theories with the negative kinetic term. The function $F(\phi, R)$, being in general nonlinear, provides a nonminimal coupling between a scalar field and curvature. Though a freedom in choosing of $F(\phi, R)$ leads to an unlimited variety of scalar-tensor theories, it is known (see, for example, [1, 2, 3]) that there exist conformal transformations transforming this kind of theories to Einstein's theory with a new minimally coupled scalar field $\phi$ and an effective potential $V(\phi)$ describing its self-interaction. The potential $V(\phi)$ is a very important ingredient of various cosmological models: a slowly

\footnotetext{
*Electronic address: sergey`sushkov@mail.ru

1 Throughout this paper we use units such that $G=c=1$. The metric signature is $(-+++)$ and the conventions for curvature tensors are $R_{\beta \gamma \delta}^{\alpha}=\Gamma_{\beta \delta, \gamma}^{\alpha}-\ldots$ and $R_{\mu \nu}=R_{\mu \alpha \nu}^{\alpha}$.
} 
varying potential behaves like an effective cosmological constat providing one or more than one inflationary phases. An appropriate choice of $V(\phi)$ is known as a problem of fine tuning of the cosmological constant.

A further extension of scalar-tensor theories can be represented by models with nonminimal couplings between derivatives of a scalar field and curvature. This kind of couplings may appear in some Kaluza-Klein theories [4, 5] (see also [6], Section 9.5). In 1993, Amendola 7] has been considered the most general gravity Lagrangian linear in the curvature scalar $R$, quadratic in $\phi$, and containing terms with four derivatives including all of the following terms (see also [8] for details):

$$
\begin{array}{cll}
L_{1}=\kappa_{1} R \phi_{, \mu} \phi^{, \mu} ; \quad L_{2}=\kappa_{2} R_{\mu \nu} \phi^{, \mu} \phi^{, \nu} ; & L_{3}=\kappa_{3} R \phi \square \phi ; \\
L_{4}=\kappa_{4} R_{\mu \nu} \phi \phi^{; \mu \nu} ; \quad L_{5}=\kappa_{5} R_{; \mu} \phi \phi^{, \mu} ; & L_{6}=\kappa_{6} \square R \phi^{2},
\end{array}
$$

where coefficients $\kappa_{1}, \ldots, \kappa_{6}$ are coupling parameters with dimensions of length-squared. Using the divergencies

$$
\left(R \phi^{, \mu} \phi\right)_{; \mu} ; \quad\left(R^{\mu \nu} \phi \phi_{, \mu}\right)_{; \nu} ; \quad\left(R^{; \mu} \phi^{2}\right)_{; \mu},
$$

one may conclude that, without loss of generality, $L_{4}, L_{5}$, and $L_{6}$ are not necessary to be considered. Also one may rule out $L_{3}$ because it contains $\phi$ itself, while coupling term of main interest are those, where only the gradient of $\phi$ is included. Thus, a general scalar-tensor theory with nonminimal derivative couplings may include only two terms $L_{1}$ and $L_{2}$.

As was shown by Amendola [7], a theory with derivative couplings cannot be recasting into Einsteinian form by a conformal rescaling $\tilde{g}_{\mu \nu}=e^{2 \omega} g_{\mu \nu}$. He also supposed that an effective cosmological constant, and then the inflationary phase can be recovered without considering any effective potential if a nonminimal derivative coupling is introduced. Amendola himself 7] has considered a cosmological model in the theory with the only derivative coupling term $L_{1}=\kappa_{1} R \phi_{, \mu} \phi^{, \mu}$ and, by using a generalized slow-rolling approximation (i.e., neglecting all terms of order higher than the second one), he has obtained some analytical inflationary solutions. A general model containing both $L_{1}$ and $L_{2}$ has been discussed in [8] (see also [9]); it was shown that the de Sitter spacetime is an attractor solution of the model if $4 \kappa_{1}+\kappa_{2}>0$. Recently Daniel and Caldwell [10] have considered a theory with the derivative coupling term $L_{2}=\kappa_{2} R_{\mu \nu} \phi^{, \mu} \phi^{, \nu}$; in particular, they studied constraints which precision tests of general relativity impose on the coupling parameter $\kappa_{2}$. It is also worth mentioning a series of papers devoted to a nonminimal modification of the Einstein-Yang-MillsHiggs theory [11] (see also a review [12] and references therein).

In this paper we continue studying a scalar-tensor theory with nonminimal derivative couplings and construct new exact cosmological solutions of the theory.

\section{FIELD EQUATIONS}

Let us consider a gravitational theory of a scalar field $\phi$ with nonminimal derivative couplings to curvature described by the action

$$
S=\int d^{4} x \sqrt{-g}\left\{\frac{R}{8 \pi}-\left[g_{\mu \nu}+\kappa_{1} g_{\mu \nu} R+\kappa_{2} R_{\mu \nu}\right] \phi^{, \mu} \phi^{, \nu}\right\} .
$$

Here coefficients $\kappa_{1}$ and $\kappa_{2}$ are derivative coupling parameters with dimensions of length-squared. Note that the action (2) does not include any potential. Varying the action (2) with respect to the metric $g_{\mu \nu}$ gives the gravitational field equations

$$
G_{\mu \nu}=8 \pi\left[T_{\mu \nu}+\kappa_{1} \Theta_{\mu \nu}^{(1)}+\kappa_{2} \Theta_{\mu \nu}^{(2)}\right]
$$

with

$$
\begin{aligned}
T_{\mu \nu}= & \nabla_{\mu} \phi \nabla_{\nu} \phi-\frac{1}{2} g_{\mu \nu}(\nabla \phi)^{2}-g_{\mu \nu} V(\phi), \\
\Theta_{\mu \nu}^{(1)}= & \nabla_{\mu} \phi \nabla_{\nu} \phi R+(\nabla \phi)^{2} G_{\mu \nu}-\nabla_{\mu} \nabla_{\nu}(\nabla \phi)^{2}+g_{\mu \nu} \square(\nabla \phi)^{2}, \\
\Theta_{\mu \nu}^{(2)}= & -\frac{1}{2} g_{\mu \nu} \nabla_{\alpha} \phi \nabla_{\beta} \phi R^{\alpha \beta}+2 \nabla_{\alpha} \phi \nabla_{(\mu} \phi R_{\nu)}^{\alpha}+\frac{1}{2} \square\left(\nabla_{\mu} \phi \nabla_{\nu} \phi\right)-\nabla_{\alpha} \nabla_{(\mu}\left(\nabla_{\nu)} \phi \nabla^{\alpha} \phi\right) \\
& +\frac{1}{2} g_{\mu \nu} \nabla_{\alpha} \nabla_{\beta}\left(\nabla^{\alpha} \phi \nabla^{\beta} \phi\right) .
\end{aligned}
$$

where $G_{\mu \nu}=R_{\mu \nu}-\frac{1}{2} g_{\mu \nu} R$ is the Einstein tensor. Then, varying the action (2) with respect to $\phi$ gives the scalar field equation of motion:

$$
g^{\mu \nu} \nabla_{\mu} \nabla_{\nu} \phi+\nabla_{\mu}\left[\nabla_{\nu} \phi\left(\kappa_{1} g^{\mu \nu} R+\kappa_{2} R^{\mu \nu}\right)\right]=0 .
$$


Note also that because of the Bianchi identity, $\nabla_{\mu} G^{\mu \nu}=0$, the scalar field and order- $\kappa$ terms form a conserved system, hence the scalar field equation (44) can be obtained as a consequence of the generalized conservation law $\nabla_{\mu}\left[T^{\mu \nu}+\kappa_{1} \Theta^{(1) \mu \nu}+\kappa_{2} \Theta^{(2) \mu \nu}\right]=0$.

Generally, the gravitational field equations (3) contain third derivatives of $\phi$, whilst the scalar field equation (4) contains third derivatives of the metric. However, an important feature of the theory (2) is the fact that the order of field equations can be reduced for a specific choice of $\kappa_{1}$ and $\kappa_{2}$. To show this we rewrite, after some algebra, the expressions for $\Theta_{\mu \nu}^{(1)}$ and $\Theta_{\mu \nu}^{(2)}$ as follows:

$$
\begin{aligned}
\Theta_{\mu \nu}^{(1)}= & \nabla_{\mu} \phi \nabla_{\nu} \phi R+(\nabla \phi)^{2} G_{\mu \nu}-2 \nabla^{\alpha} \phi \nabla^{\beta} \phi R_{\mu \alpha \nu \beta}-2 \nabla_{\mu} \nabla^{\alpha} \phi \nabla_{\nu} \nabla_{\alpha} \phi-2 \nabla^{\alpha} \phi \nabla_{\alpha} \nabla_{\mu} \nabla_{\nu} \phi \\
& +2 g_{\mu \nu}\left[\nabla^{\alpha} \nabla^{\beta} \phi \nabla_{\alpha} \nabla_{\beta} \phi+\nabla_{\alpha} \phi \nabla_{\beta} \phi R^{\alpha \beta}+\nabla^{\alpha} \phi \nabla_{\alpha} \square \phi\right], \\
\Theta_{\mu \nu}^{(2)}= & 2 \nabla_{\alpha} \phi \nabla_{(\mu} \phi R_{\nu)}^{\alpha}-\nabla_{\mu} \nabla_{\nu} \phi \square \phi-\nabla^{\alpha} \phi \nabla_{\alpha} \nabla_{\mu} \nabla_{\nu} \phi \\
& +\frac{1}{2} g_{\mu \nu}\left[\nabla^{\alpha} \nabla^{\beta} \phi \nabla_{\alpha} \nabla_{\beta} \phi+(\square \phi)^{2}+2 \nabla^{\alpha} \phi \nabla_{\alpha} \square \phi\right] .
\end{aligned}
$$

It is seen that both expressions contain similar third-order terms, $\nabla^{\alpha} \phi \nabla_{\alpha} \nabla_{\mu} \nabla_{\nu} \phi$ and $g_{\mu \nu} \nabla^{\alpha} \phi \nabla_{\alpha} \square \phi$, which are cancelled in the combination $\kappa_{1} \Theta_{\mu \nu}^{(1)}+\kappa_{2} \Theta_{\mu \nu}^{(2)}$ provided the coupling parameters are chosen as follows

$$
-2 \kappa_{1}=\kappa_{2} .
$$

Hereafter we will assume that the relation (77) hold. Denoting $\kappa=-2 \kappa_{1}=\kappa_{2}$, we can rewrite the action (2) as follows

$$
S=\int d^{4} x \sqrt{-g}\left\{\frac{R}{8 \pi}-\left[g_{\mu \nu}+\kappa G_{\mu \nu}\right] \phi^{, \mu} \phi^{, \nu}\right\} .
$$

The gravitational field equations (3) now read

$$
G_{\mu \nu}=8 \pi\left[T_{\mu \nu}+\kappa \Theta_{\mu \nu}\right]
$$

with

$$
\begin{aligned}
\Theta_{\mu \nu}= & -\frac{1}{2} \Theta_{\mu \nu}^{(1)}+\Theta_{\mu \nu}^{(2)} \\
= & -\frac{1}{2} \nabla_{\mu} \phi \nabla_{\nu} \phi R+2 \nabla_{\alpha} \phi \nabla_{(\mu} \phi R_{\nu)}^{\alpha}-\frac{1}{2}(\nabla \phi)^{2} G_{\mu \nu}+\nabla^{\alpha} \phi \nabla^{\beta} \phi R_{\mu \alpha \nu \beta}+\nabla_{\mu} \nabla^{\alpha} \phi \nabla_{\nu} \nabla_{\alpha} \phi \\
& -\nabla_{\mu} \nabla_{\nu} \phi \square \phi+g_{\mu \nu}\left[-\frac{1}{2} \nabla^{\alpha} \nabla^{\beta} \phi \nabla_{\alpha} \nabla_{\beta} \phi+\frac{1}{2}(\square \phi)^{2}-\nabla_{\alpha} \phi \nabla_{\beta} \phi R^{\alpha \beta}\right],
\end{aligned}
$$

and the scalar field equation of motion (4) yields

$$
\left[g^{\mu \nu}+\kappa G^{\mu \nu}\right] \nabla_{\mu} \nabla_{\nu} \phi=0 .
$$

Let us emphasize once more that the field equations (9) and (10) contain now only second derivatives of $g_{\mu \nu}$ and $\phi$. Thus, from the physical point of view, the theory (8) can be interpreted as a "good" dynamical theory.

\section{COSMOLOGICAL MODELS}

Consider a spatially-flat cosmological model with a metric

$$
d s^{2}=-d t^{2}+e^{2 \alpha(t)} d \mathbf{x}^{2},
$$

where $a(t)=e^{\alpha(t)}$ is the scale factor, and $d \mathbf{x}^{2}$ is the Euclidian metric, and assume that $\phi=\phi(t)$. In this case the field equations (9) and (10) are reduced to the following system:

$$
\begin{gathered}
3 \dot{\alpha}^{2}=4 \pi \dot{\phi}^{2}\left(1-9 \kappa \dot{\alpha}^{2}\right), \\
-2 \ddot{\alpha}-3 \dot{\alpha}^{2}=4 \pi \dot{\phi}^{2}\left[1+\kappa\left(2 \ddot{\alpha}+3 \dot{\alpha}^{2}+4 \dot{\alpha} \ddot{\phi} \dot{\phi}^{-1}\right)\right], \\
\ddot{\phi}+3 \dot{\alpha} \dot{\phi}-3 \kappa\left[\dot{\alpha}^{2} \ddot{\phi}+2 \dot{\alpha} \ddot{\alpha} \dot{\phi}+3 \dot{\alpha}^{3} \dot{\phi}\right]=0 .
\end{gathered}
$$

where a dot means a derivative with respect to time. Note that Eqs. (13) and (14) are of second order, while Eq. (12) is a first-order differential constraint for $\alpha(t)$ and $\phi(t)$. 
First, let us discuss the simple case $\kappa=0$, which just means the absence of derivative coupling. In this case Eqs. (12)-(14) are easily solved resulting in

$$
\begin{aligned}
& \alpha(t)=\alpha_{0}+\frac{1}{3} \ln \left(t-t_{0}\right), \\
& \phi(t)=\phi_{0}+\frac{1}{2 \sqrt{3 \pi}} \ln \left(t-t_{0}\right),
\end{aligned}
$$

where $t_{0}, \alpha_{0}$ and $\phi_{0}$ are constants of integration. Without loss of generality one may put $\alpha_{0}=0$ and $\phi_{0}=0$, then the corresponding metric reads

$$
d s^{2}=-d t^{2}+\left(t-t_{0}\right)^{2 / 3} d \mathbf{x}^{2} .
$$

The spacetime with the metric (17) has an initial singularity at $t=t_{0}$.

Consider now a general case $\kappa \neq 0$. In this case the constraint (12) can be rewritten as follows

$$
\dot{\phi}^{2}=\frac{3 \dot{\alpha}^{2}}{4 \pi\left(1-9 \kappa \dot{\alpha}^{2}\right)}
$$

or, equivalently,

$$
\dot{\alpha}^{2}=\frac{4 \pi \dot{\phi}^{2}}{3\left(1+12 \pi \kappa \dot{\phi}^{2}\right)} .
$$

From here it follows that $\dot{\alpha}$ and $\dot{\phi}$ should obey the following conditions:

$$
\begin{gathered}
1-9 \kappa \dot{\alpha}^{2}>0, \\
1+12 \pi \kappa \dot{\phi}^{2}>0 .
\end{gathered}
$$

Let us now separate equations for $\alpha$ and $\phi$. For this aim, we resolve Eqs. (13) and (14) with respect to $\ddot{\alpha}$ and $\ddot{\phi}$ and, using the relations (18) and (19), eliminate $\dot{\phi}$ and $\dot{\alpha}$ from respective equations. As the result, we find

$$
\begin{gathered}
\ddot{\alpha}=-\frac{3 \dot{\alpha}^{2}\left(1-3 \kappa \dot{\alpha}^{2}\right)\left(1-9 \kappa \dot{\alpha}^{2}\right)}{1-9 \kappa \dot{\alpha}^{2}+54 \kappa^{2} \dot{\alpha}^{4}} \\
\ddot{\phi}=-\frac{2 \sqrt{3 \pi} \dot{\phi}^{2}\left(1+8 \pi \kappa \dot{\phi}^{2}\right)\left(1+12 \pi \kappa \dot{\phi}^{2}\right)^{1 / 2}}{1+12 \pi \kappa \dot{\phi}^{2}+96 \pi^{2} \kappa^{2} \dot{\phi}^{4}} .
\end{gathered}
$$

Since $\dot{\alpha}$ and $\dot{\phi}$ obey the conditions (20) and (21), it is seen that $\ddot{\alpha}$ and $\ddot{\phi}$ are negative for all times. In turns, this means that $\dot{\alpha}$ and $\dot{\phi}$ are monotonically decreasing with time.

Let us analyze an asymptotical behavior of $\alpha$ and $\phi$ for large times. Suppose that $\dot{\alpha}$ tends to some nonzero constant at $t \rightarrow \infty$; respectively, this means that $\ddot{\alpha}$ should go to zero. However, as it follows from Eq. (22), $\ddot{\alpha}$ is not zero in this limit. Thus, we face with a contradiction and, therefore, should conclude that $\dot{\alpha} \rightarrow 0$ if $t \rightarrow \infty$. By using this asymptotical property, we obtain the following asymptotic form of Eq. (22):

$$
\ddot{\alpha} \approx-3 \dot{\alpha}^{2} .
$$

The corresponding asymptotical solution is

$$
\alpha_{t \rightarrow \infty}=\alpha_{\infty}+\frac{1}{3} \ln \left(t-t_{\infty}\right),
$$

where $t_{\infty}$ and $\alpha_{\infty}$ are constants of integration. An asymptotic for $\phi$ can be found straightforwardly from the constraint (18):

$$
\phi_{t \rightarrow \infty}=\phi_{\infty}+\frac{1}{2 \sqrt{3 \pi}} \ln \left(t-t_{\infty}\right)
$$

where $\phi_{\infty}$ is a constant of integration. It is worth noting that the asymptotics (25) and (26) do not depend on $\kappa$ and coincide with exact solutions (15) and (16) obtained for $\kappa=0$. 


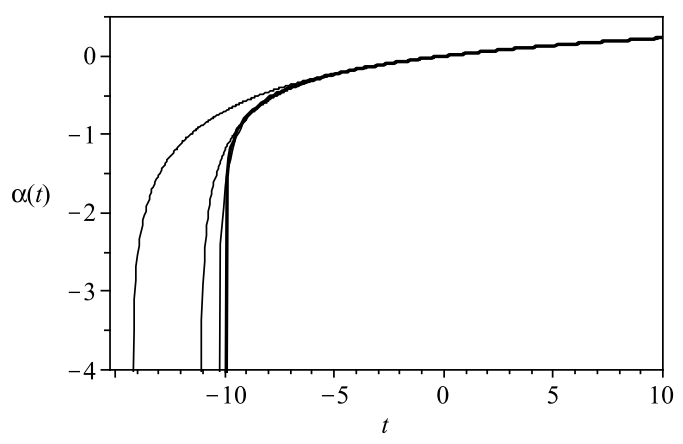

FIG. 1: Plots of $\alpha(t)$ for negative $\kappa=-1 ;-10 ;-100$ (from right to left). The thick curve corresponds to $\kappa=0$ (no derivative coupling).

To characterize an asymptotical behavior of $\alpha$ and $\phi$ for small times, we consider separately two cases. First, let $\kappa$ be negative, $\kappa<0$. In this case the condition (21) gives the following bound for $\dot{\phi}$ :

$$
\dot{\phi}^{2}<\frac{1}{12 \pi|\kappa|},
$$

while the condition (20) is fulfilled for all values of $\dot{\alpha}$. Since $\dot{\alpha}$ is monotonically decreasing with time, its value should be growing with decreasing time. Let $t_{i}$ be some initial moment of time (possibly $t_{i}=-\infty$ ). Suppose that $\dot{\alpha}$ tends to some constant value in the limit $t \rightarrow t_{i}$; respectively, this means that $\ddot{\alpha}$ should go to zero. However, as follows from Eq. (22), $\ddot{\alpha}$ is not zero in this limit. This is a contradiction, and hence we should conclude that $\dot{\alpha}$ is boundlessly increasing in the limit $t \rightarrow t_{i}$. Assuming $\dot{\alpha} \rightarrow \infty$ at $t \rightarrow t_{i}$ gives the following asymptotical form of Eq. (22):

$$
\ddot{\alpha} \approx-\frac{3}{2} \dot{\alpha}^{2}
$$

with the asymptotic solution

$$
\alpha_{t \rightarrow t_{i}}=\alpha_{i}+\frac{2}{3} \ln \left(t-t_{i}\right)
$$

The asymptotic for $\phi$ is found from Eq. (18) as

$$
\phi_{t \rightarrow t_{i}}=\phi_{i}+\frac{t}{2 \sqrt{3 \pi|\kappa|}}
$$

where $t_{i}, \alpha_{i}$ and $\phi_{i}$ are constants of integration. The corresponding asymptotical form of the metric (11) is

$$
d s_{t \rightarrow t_{i}}^{2}=-d t^{2}+e^{2 \alpha_{i}}\left(t-t_{i}\right)^{4 / 3} d \mathbf{x}^{2} .
$$

A spacetime with this metric is singular at $t=t_{i}$. This singularity is analogous to initial cosmological singularities in models with usual scalar fields. However, a new interesting feature of the examined model is that the scalar field with negative derivative coupling $\kappa<0$ has the regular behavior (30) near the singularity.

Results of numerical study of Eq. (22) in case $\kappa<0$ are shown in Fig. 1. Obtained solutions reproduce all asymptotical properties found above analytically.

Then, let $\kappa$ be positive, $\kappa>0$. In this case the condition (20) gives the following bound for $\dot{\alpha}$ :

$$
\dot{\alpha}^{2}<\frac{1}{9 \kappa}
$$

while Eq. (21) is fulfilled for any $\dot{\phi}$. Repeating the above arguments, we may conclude that $\dot{\phi} \rightarrow \infty$ at $t \rightarrow-\infty$. This gives the following asymptotic form of Eq. (23):

$$
\ddot{\phi} \approx-\frac{\dot{\phi}}{\sqrt{\kappa}}
$$




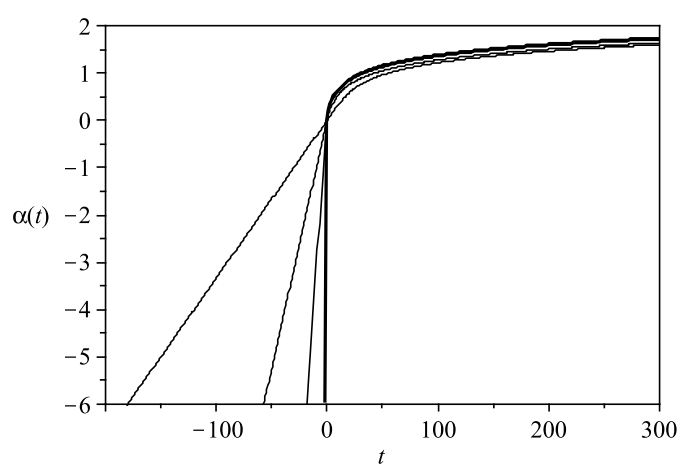

FIG. 2: Plots of $\alpha(t)$ for positive $\kappa=1 ; 10 ; 100$ (from right to left). The thick curve corresponds to $\kappa=0$ (no derivative coupling).

with the asymptotic solution

$$
\phi_{t \rightarrow-\infty}=\phi_{2}+C e^{-t / \sqrt{\kappa}}
$$

where $\phi_{2}$ and $C$ are constants of integration. To obtain an asymptotic for $\alpha$ one may substitute Eq. (34) into (19) and find after some algebra

$$
\alpha \approx \frac{t-t_{0}}{3 \sqrt{\kappa}}-\frac{e^{2 t / \sqrt{\kappa}}}{144 \pi \kappa C^{2}}
$$

where $t_{0}$ is a constant of integration which without loss of generality can be set zero. We see that in the limit $t \rightarrow-\infty$ the scalar $\phi$ is exponentially growing, and $\alpha$ is exponentially approximating to its asymptotic

$$
\alpha_{t \rightarrow-\infty}=\frac{t}{3 \sqrt{\kappa}} .
$$

Hence, in the limit $t \rightarrow-\infty$ the spacetime metric (11) takes asymptotically the de Sitter-like form:

$$
d s_{t \rightarrow-\infty}^{2}=-d t^{2}+e^{2 H t} d \mathbf{x}^{2},
$$

with $H=(3 \sqrt{\kappa})^{-1}$. Thus, in the case $\kappa>0$ an universe at early stages has the quasi-de Sitter behavior corresponding to the cosmological constant $\Lambda=3 H^{2}=(3 \kappa)^{-1}$.

Results of numerical study in the case $\kappa>0$ are shown in Fig. 2. Note that all $\kappa>0$ solutions represent an interesting feature. Namely, they describe two phases in evolution of the universe. First, for an infinitely long time the universe is living in the quasi-de Sitter or inflationary phase. Then, during a relatively short time the universe exits from the inflationary stage and goes to a power-law expansion with $a(t) \sim t^{1 / 3}$ (it is worth noting that this law corresponds to the equation of state $p=\rho$ ).

\section{CONCLUSIONS}

We have considered the gravitational theory of a scalar field with nonminimal derivative coupling to curvature and studied cosmological models in this theory. The main results obtained are as follows:

1. The Lagrangian of the theory includes two derivative coupling terms $\kappa_{1} R \phi_{, \mu} \phi^{, \mu}$ and $\kappa_{2} R_{\mu \nu} \phi^{, \mu} \phi^{, \nu}$, where $\kappa_{1}$ and $\kappa_{2}$ are coupling parameters with dimensions of length-squared. In general, field equations of the theory are of third order, i.e., contain third derivatives of $g_{\mu \nu}$ and $\phi$, but in the particular case the order of equations is reduced up to the second one. This case corresponds to the choice $-2 \kappa_{1}=\kappa_{2} \equiv \kappa$, then a combination of derivative coupling terms turn into $\kappa G_{\mu \nu} \phi^{, \mu} \phi^{, \nu}$. It is worth noting that Capozziello et al [8] , at pages 43 and 47 , have mentioned the case $-2 \kappa_{1}=\kappa_{2}$ to play a special role, because it represents a singular point of the differential equation. In this paper, we have supposed that the theory with $-2 \kappa_{1}=\kappa_{2}$ is more preferable with the physical point of view, since the corresponding field equations do not contain derivatives of dynamical variables of order higher than the second.

2. Assuming $-2 \kappa_{1}=\kappa_{2} \equiv \kappa$, we have studied a cosmological model with the spatially-flat FriedmanRobertson-Walker metric. It was shown that a behavior of the scale factor $a(t)$ and the scalar field $\phi$ at large 
times is the same for all values of $\kappa$ including zero, that is the late evolution of universe does not depend on $\kappa$. Namely, one has $a(t) \sim t^{1 / 3}$ and $\phi(t) \sim \ln t$ at $t \rightarrow \infty$. Note this asymptotical behavior coincides with that of the exact solution (15), (16) obtained for $\kappa=0$ (no coupling).

3. General properties of the model crucially depends on a sign of $\kappa$. For $\kappa<0$ an asymptotical form of the cosmological metric for small times is given by Eq. (31). A corresponding scale factor is $a(t) \sim$ $\left(t-t_{i}\right)^{2 / 3}$; it describes the universe with an initial singularity at $t=t_{i}$. A new interesting feature of the model with derivative coupling is that a behavior of the scalar field near the cosmological singularity is regular, $\phi(t) \sim t$ (see Eq. (30) $)$. For $\kappa>0$ the law of universe evolution is qualitatively distinct from that for $\kappa<0$. Now at early stages the universe has the quasi-de Sitter behavior (37) corresponding to the cosmological constant $\Lambda=(3 \kappa)^{-1}$. In the limit $t \rightarrow-\infty$ the scale factor has the following asymptotical

form $a(t) \sim \exp \left(\frac{t}{3 \kappa^{1 / 2}}-\frac{e^{2 t / \sqrt{\kappa}}}{144 \pi \kappa C^{2}}\right)$ (see Eq. (35)), hence $a(t)$ exponentially fast goes to the de-Sitter form $a(t)=e^{H t}$ with $H=(3 \sqrt{\kappa})^{-1}$. At the same time, the scalar field $\phi$ is exponentially growing at $t \rightarrow-\infty$, namely $\phi(t) \sim e^{-t / \sqrt{\kappa}}$.

In conclusion, let us summarize the most essential features of cosmology with nonminimal derivative coupling. First of all, we should emphasize that cosmological solutions with the quasi-de Sitter phase are typical solutions of the gravitational theory of a scalar field with derivative coupling of the form $\kappa G_{\mu \nu} \phi^{, \mu} \phi^{, \nu}$ with positive $\kappa$. So, in order to obtain an inflationary phase, one need no fine-tuned potential, and so one do not face with the problem of fine-tuning. Another important feature of the model consists in the fact that an exact cosmological solution with $\kappa>0$ describes in a unique manner both a quasi-de Sitter phase and an exit from it. Thus, the problem of graceful exit from inflation in cosmology with the derivative coupling term $\kappa G_{\mu \nu} \phi^{, \mu} \phi^{, \nu}$ has a natural solution without any fine-tuned potential.

\section{Acknowledgments}

This work was supported in part by the Russian Foundation for Basic Research grants No. 08-02-91307, 08-02-00325.

[1] K. Maeda, Phys. Rev. D39, 3159 (1989).

[2] V. Faraoni, E. Gunzig, P. Nardone, Fund.CosmicPhys. 20, 121 (1999).

[3] J.L. Crooks, P.H. Frampton, Phys. Rev. D73, 123512 (2006).

[4] Q. Shafi and C. Wetterich, Phys. Lett. 152B, 51 (1985).

[5] Q. Shafi and C. Wetterich, Nucl. Phys. B289, 787 (1987).

[6] A. Linde, Particle Physics and Inflationary Cosmology, (1990) Harwood Publ. London.

[7] L. Amendola, Phys.Lett. B301 (1993) 175; arXiv:gr-qc/9302010 [gr-qc].

[8] S. Capozziello, G. Lambiase, H.-J.Schmidt, Annalen Phys. 9 (2000) 39; arXiv:gr-qc/9906051 [gr-qc].

[9] S. Capozziello, G. Lambiase, Gen.Rel.Grav. 31 (1999) 1005; arXiv:gr-qc/9901051 [gr-qc].

[10] S.F. Daniel and R. Caldwell, Class.Quant.Grav. 24, 5573 (2007); arXiv:0709.0009 [gr-qc].

[11] A.B. Balakin, H. Dehnen, A.E. Zayats, Phys.Rev.D76 (2007) 124011; arXiv:0710.5070 [gr-qc]; Gen.Rel.Grav.40 (2008) 2493; arXiv:0803.3442 [gr-qc]; Int.J.Mod.Phys.D17 (2008) 1255; arXiv:0710.4992 [gr-qc].

[12] A.B. Balakin, H. Dehnen, A.E. Zayats, Annals Phys. 323 (2008) 2183; arXiv:0804.2196 [gr-qc]. 